Orientierung der Schnittebenen wurden bei zwei verschiedenen Untersuchungen neue Läsionen gesehen, wo keine waren, und damit eine zeitliche Streuung diagnostiziert. „Es gibt eine Überdiagnose von MS-Läsionen und eine Unterdiagnose von MSSymptomen“, sagte Lebrun-Frénay.

Die Neurologin gab sich jedoch offen für eine Therapie bei RIS, sofern klar sei, dass sich damit eine MS verhindern lasse. Dies werde derzeit in den beiden Studien ARISE mit Dimethylfumarat und TERIS mit Teriflunomid geprüft. Ferner müsse genauer untersucht werden, welche Patienten das höchste Konversionsrisiko tragen. Möglicherweise lasse sich dann eine Therapie bei solchen Hochrisikopatienten rechtfertigen. Um das Konversionsrisiko und die entscheidenden Risikofaktoren $\mathrm{zu}$ ermitteln, müssten jedoch RIS-Kohorten über zehn bis 15 Jahre hinweg untersucht werden, bislang gebe es allenfalls brauchbare Daten für einen 5-Jahres-Zeitraum, und da seien die Konversionsraten in Studien deutlich geringer als bei CIS-Patienten. „Es gibt also noch viel zu tun, bevor wir bei RIS eine Therapie empfehlen können“, so Lebrun-Frénay. Dem stimmten auch die meisten Teilnehmer im Vortragssaal zu. Nur eine Minderheit favorisierte in der Abstimmung im Anschluss an die Diskussion jetzt schon die MS-Therapie bei einem RIS.

Thomas Müller, Springer Medizin

Hot Topic Session 3: RIS: should it be treated? $7^{\text {th }}$ Joint ECTRIMS-ACTRIMS

Meeting, Paris, 25. - 28.10.2017

\title{
MS in der Kindheit, kognitive Probleme als Erwachsener
}

\begin{abstract}
Beginnt eine MS in der Kindheit, hat sie gravierendere Auswirkungen auf das Gehirn als bei Erwachsenen. So kommt es offenbar schneller zu kognitiven Problemen als bei einem späteren Krankheitsbeginn.
\end{abstract}

Der Tod von Axonen und Nervenzellen führt bei den meisten MS-Patienten im Krankheitsverlauf zu kognitiven Problemen. Tritt eine MS bei Kindern und Jugendlichen auf, könnten die Effekte noch gravierender sein als bei Erwachsenen, da die Gehirnentwicklung noch nicht abgeschlossen ist. Andererseits ist bei Minderjährigen die Regenerationsfähigkeit höher, möglicherweise können sie die negativen Auswirkungen einer MS besser kompensieren. Was letztlich zutrifft, lässt sich bislang nicht sagen, die Literatur dazu ist sehr widersprüchlich. Immerhin gebe es Hinweise, dass Patienten, deren MS in der Kindheit begann, in Kognitionstests schlechter abschnitten als Erwachsene mit vergleichbarer Krankheitsdauer, aber einem Beginn im Erwachsenenalter, erläuterte Dr. Luis Ruano von der Universität in Porto. Der MS-Experte hat zusammen mit italienischen Neurologen in einer eigenen Querschnittstudie nach weiteren Hinweisen gesucht. Danach scheint sich ein MS-Beginn in der Kindheit oder Jugend (pediatric onset, POMS) deutlich stärker auf die kognitiven Fähigkeiten auszuwirken als ein MS-Beginn im Erwachsenenalter (adult onset, AOMS).

\section{Gehirn arbeitet langsamer}

Die Wissenschaftler haben Angaben aus sechs MS-Zentren in Italien ausgewertet. Sie verglichen die kognitive Leistung bei 119 Patienten mit POMS und 712 Patienten mit AOMS. Zum Zeitpunkt der Analyse waren alle Patienten bereits erwachsen, das Alter derjenigen mit POMS betrug im Schnitt 30 Jahre, die Krankheitsdauer rund 13 Jahre. Patienten mit AOMS waren im Schnitt 42 Jahre alt und seit neun Jahren krank. Unterschiede gab es auch beim EDSS-Wert: Dieser hatte bei Patienten mit AOMS einen Wert von 2,0 erreicht, bei denen mit POMS einen Wert von 2,5. Sämtliche Teilnehmer unterzogen sich einer Batterie von Kognitionstests, außerdem bestimmten die Studienärzte das Ausmaß von Fatigue und Depressionen. Insgesamt schnitten die Patienten mit POMS in den Kognitionstests nicht schlech-
T1 Vergleich der Patienten mit MS mit pädiatrischem Beginn der Erkrankung (pediatric onset, POMS) und Beginn im Erwachsenenalter (AOMS)

\begin{tabular}{llll}
\hline & $\begin{array}{l}\text { AOMS } \\
(\mathrm{n}=119)\end{array}$ & $\begin{array}{l}\text { POMS } \\
(\mathrm{n}=119)\end{array}$ & $\mathrm{p}$-Wert \\
Krankheitsdauer (Median Jahre) & 12,3 & 13,2 & n. S. \\
Alter (Median Jahre) & 34,9 & 27,7 & n. S. \\
EDSS (Median Score) & 2,5 & 2,5 & n. s. \\
Geschlecht (\% weiblich) & 67,7 & 73,0 & n. S. \\
Kognitive Beeinträchtigung & $28,7 \%$ & $44,5 \%$ & 0,02 \\
( $\geq 2$ Domänen & & & \\
Nach Vortrag Dr. Luis Ruano, Universität Porto, ECTRIMS 2017 & \\
\hline
\end{tabular}

ter ab, allerdings waren sie im Mittel auch zwölf Jahre jünger. Wurde das Alter berücksichtigt, zeigten sie deutlich häufiger eine unterdurchschnittliche Leistung in mindestens zwei kognitiven Domänen als Patienten mit AOMS: Der Unterschied betrug rund $70 \%$ und ließ sich vor allem auf Defizite bei der Verarbeitungsgeschwindigkeit zurückführen.

\section{Junge Gehirne besonders gefährdet?}

Da Patienten mit POMS länger krank waren und bereits mehr Behinderungen akkumuliert hatten als solche mit AOMS, könnte auch dies die schlechtere Leistung erklären. Verglichen die Forscher jedoch nur Patienten mit gleichem EDSS-Wert und ähnlicher Krankheitsdauer, zeigten sich wiederum deutlich Unterschiede: So hatten von den Patienten mit POMS 45\% kognitive Defizite, $29 \%$ waren es bei den Betroffenen mit AOMS, und dies, obwohl Letztere im Schnitt etwas älter waren (35 vs. 28 Jahre) ( Tab. 1). Auch hier zeigten sich vor allem Unterschiede bei der Verarbeitungsgeschwindigkeit. Keine Differenzen gab es bei Fatigue- und Depressionswerten. Möglicherweise reagiert ein junges Gehirn also doch empfindlicher auf die MS als das eines Erwachsenen.

Thomas Müller, Springer Medizin

Free Communications 4: L. Ruano L: Patients with pediatric-onset multiple sclerosis are at higher risk of cognitive impairment in adulthood: an Italian collaborative study. $7^{\text {th }}$ Joint ECTRIMS-ACTRIMS Meeting, Paris, 25. - 28.10.2017 